\title{
Design of a chiral elastic structure supporting interfacial waveforms
}

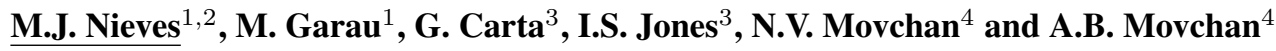 \\ ${ }^{1}$ Keele University, School of Computing and Mathematics, Keele, UK \\ ${ }^{2}$ University of Cagliari, Department of Mechanical, Chemical and Material Engineering, Cagliari, Italy \\ ${ }^{3}$ Liverpool John Moores University, Mechanical Engineering and Materials Research Centre, Liverpool, \\ UK \\ ${ }^{4}$ University of Liverpool, Department of Mathematical Sciences, Liverpool, UK \\ m.nieves@keele.ac.uk
}

\begin{abstract}
An infinite heterogeneous elastic triangular lattice connected to a non-uniform array of gyroscopic spinners is considered. An algorithm is described for generating interfacial waves that propagate along the boundaries of subdomains containing inhomogeneities in the spinner array. The interfacial waveforms have preferential directions that can be controlled through adjusting the frequency of excitation or the arrangement of the spinners.
\end{abstract}

\section{INTRODUCTION}

Structured systems composed of fundamental mechanical elements can be easily designed and they can produce a broad range of extraordinary dynamic phenomena. We consider a triangular elastic structured system possessing chirality. The latter is imposed by attaching the elastic system to an array of gyroscopic spinners (See Figure 1(a)). The first model of this type appeared in [1], where a uniform chiral lattice was shown to suppress pressure waves and was used to design an efficient cloaking and shielding device. The dispersive nature of this system was further studied in [2]. Extensions of [1] have appeared in [3], where designs for robust chiral topological insulators that support edge modes were studied. Experimental evidence of edge modes in finite gyroscopic systems have been presented in [4]. In the high-frequency regime, an elastic medium connected to a non-uniform array of spinners has been shown to admit exponentially localised Gaussian beams, whose paths can be controlled [5].

Recently, a hexagonal structure attached to gyroscopic spinners was investigated in [6]. It was shown that if the spinner array is heterogeneous, one can generate uni-directional localised interfacial waveforms with preferential directions that propagate along the internal boundaries of the system. The effect of introducing soft internal links into the hexagonal system, to create a heterogeneous chiral triangular lattice, was also studied. Here, we demonstrate that interfacial waveforms can be excited in an inhomogeneous chiral triangular lattice that follow a closed path and have preferential directions.

\section{Model of AN INFINITE INHOMOGENEOUS ELASTIC LATTICE CONNECTED TO SPINNERS}

We consider an inhomogeneous triangular lattice, arranged as in Figure 1(a), with elastic links connecting periodically placed masses at the junctions. The lattice contains links of length $l$, having stiffness $\mu$ (the black lines of Figure 1(a)) or $\mu_{\varepsilon}$ (the grey lines). We define $\varepsilon=\mu_{\varepsilon} / \mu$, representing the contrast in the stiffnesses and assume $\varepsilon<1$. Each node has mass $m$ and is assumed to be connected to a gyroscopic spinner shown in Figure 1(b). The effect brought by the spinners has been modelled in [1], assuming the angle of nutation $\theta$ is small. The spinners introduce terms that couple the in-plane displacement components of the masses in the governing equations. The strength of the coupling is governed by the parameter $\alpha$, known as the spinner constant. The lattice elementary cell is defined by the basis vectors $\mathbf{t}^{(1)}=(3 l, 0)^{\mathrm{T}}$ and $\mathbf{t}^{(2)}=(3 l / 2, \sqrt{3} l / 2)^{\mathrm{T}}$, contains 3 masses and is associated with the multi-index $\mathbf{n}=\left(n_{1}, n_{2}\right)^{\mathrm{T}}, n_{j} \in \mathbb{Z}, j=1,2$. In the time-harmonic regime, masses vibrate with a radian frequency $\omega$ and displacement amplitudes represented by the vectors $\mathbf{u}^{(\mathbf{n})}, \mathbf{v}^{(\mathbf{n})}$ and $\mathbf{w}^{(\mathbf{n})}$.

We look for the existence of Floquet-Bloch waves whose periodicity is defined according to the elementary cell presented in Figure 1(a). By introducing the vector $\mathbf{U}^{(\mathbf{n})}=\left(\left(\mathbf{u}^{(\mathbf{n})}\right)^{\mathrm{T}},\left(\mathbf{v}^{(\mathbf{n})}\right)^{\mathrm{T}},\left(\mathbf{w}^{(\mathbf{n})}\right)^{\mathrm{T}}\right)^{\mathrm{T}}$, the governing 


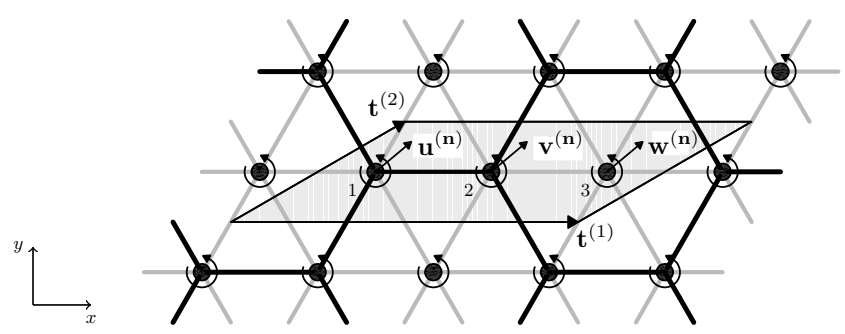

(a)

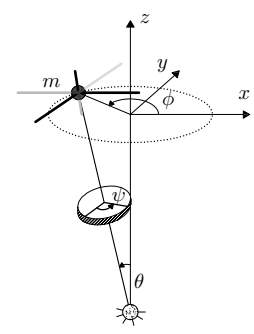

(b)

Fig. 1: (a) An inhomogeneous triangular lattice linked to a uniform system of spinners and (b) a representative gyroscopic spinner, where $\psi, \phi$ and $\theta$ are the angles of spin, precession and nutation respectively. The directions of spin possessed by the spinners are indicated by the circular arrows in (a).

equations for a single elementary cell of the chiral elastic lattice can be written in the condensed form

$$
\left[\mathbf{C}_{\varepsilon}(\mathbf{k})-\omega^{2}(\mathbf{M}-\mathbf{R})\right] \mathbf{U}^{(\mathbf{n})}=\mathbf{0}
$$

where $\mathbf{k}=\left(k_{1}, k_{2}\right)^{\mathrm{T}}$ is the Bloch vector. In the above, $\mathbf{M}=m \mathbf{I}_{6}$ is a mass matrix for the elementary cell, with $\mathbf{I}_{j}$ being the $j \times j$ identity matrix. The matrices $\mathbf{R}$ and $\mathbf{C}_{\varepsilon}$ represent the contribution due to the spinners and the stiffness of the system, respectively. They are

$$
\mathbf{R}=\mathrm{i} \alpha \operatorname{diag}\left(\left(\begin{array}{rr}
0 & -1 \\
1 & 0
\end{array}\right),\left(\begin{array}{rr}
0 & -1 \\
1 & 0
\end{array}\right),\left(\begin{array}{rr}
0 & -1 \\
1 & 0
\end{array}\right)\right) \quad \text { and } \quad \mathbf{C}_{\varepsilon}(\mathbf{k})=\frac{\mu}{4}\left(\begin{array}{cc}
\mathbf{C}_{\varepsilon}^{(1)} & \mathbf{C}_{\varepsilon}^{(2)} \\
\mathbf{C}_{\varepsilon}^{(2) \mathrm{T}} & 12 \varepsilon \mathbf{I}_{2}
\end{array}\right)
$$

Here, the stiffness matrix has the submatrices

$$
\mathbf{C}_{\varepsilon}^{(1)}=\left(\begin{array}{cc}
6(1+\varepsilon) \mathbf{I}_{2} & \left.\mathbf{D}^{(1)}(\kappa)\right|_{\kappa=0}+\mathbf{D}^{(2)} \\
\left.\hline\left.\mathbf{D}^{(1)}(\kappa)\right|_{\kappa=0}+\mathbf{D}^{(2)}\right]^{\mathrm{T}} & 6(1+\varepsilon) \mathbf{I}_{2}
\end{array}\right), \quad \mathbf{C}_{\varepsilon}^{(2)}=\varepsilon\left(\begin{array}{c}
\mathbf{D}^{(1)}(\kappa)-\mathbf{D}^{(2)} \\
\left.\mathbf{D}^{(1)}(\kappa)\right|_{\kappa=0}+\mathbf{D}^{(2)}
\end{array}\right),
$$

where

$$
\mathbf{D}^{(1)}(\kappa)=-\left(\begin{array}{cc}
4 \mathrm{e}^{-\mathrm{i} \kappa}+\xi_{+} & 0 \\
0 & 3 \xi_{+}
\end{array}\right), \quad \mathbf{D}^{(2)}=\sqrt{3} \xi_{-}\left(\begin{array}{cc}
0 & 1 \\
1 & 0
\end{array}\right)
$$

$\xi_{ \pm}=\mathrm{e}^{-\mathrm{i} \eta} \pm \mathrm{e}^{-\mathrm{i} \gamma}, \kappa=3 k_{1} l, \eta=\left(3 k_{1}-\sqrt{3} k_{2}\right) l / 2$ and $\gamma=\left(3 k_{1}+\sqrt{3} k_{2}\right) l / 2$. The non-trivial solutions of system (1) determine the eigenfrequencies and corresponding eigenmodes for free vibrations in the lattice.

In Figure 2(a), a representative dispersion diagram for the case of $\varepsilon=0.2$ and $\alpha=0.7$ is presented. All other material parameters are set to unity. There, we can see six dispersion curves and four finite stop bands. The highest finite stop band reduces in size if the spinner constant $\alpha$ approaches unity from below. In this case, a Dirac cone appears on the boundary of the Brillouin zone at K. For $\alpha=0.7$, the highest stop band in Figure 2(a) represents a perturbation from this configuration.

\section{EXAMPLE: INTERFACIAL WAVEFORMS FOR INHOMOGENEOUS CHIRAL ELASTIC STRUCTURES}

Now we consider an inhomogeneous triangular structure attached to a heterogeneous array of spinners, referred to here as the inhomogeneous chiral elastic lattice. We assume the array has a subset in which the properties of the spinners are different from those in the ambient lattice. The boundary of this subset defines the internal boundary of the system. The lattice is excited by a time harmonic displacement with the form $\mathbf{A} e^{\mathrm{i} \omega t}$, where $|\mathbf{A}|=1$ is the amplitude and $t$ represents time. The response of the system is determined using Comsol Multiphysics (version 5.2a). To simulate an infinite lattice, we attach PML to the sides of the computational geometry.

A rectangle, with dimensions $90 l \times 76.2 l,(l=1)$, representing the inhomogeneous chiral elastic lattice was programmed. The internal boundary of the system is represented by an equilateral triangle of side length $55 l$. The spinners located inside this region possess a spinner constant $\alpha=-0.7$, while those outside this set have $\alpha=0.7$. The inhomogeneity within the elastic lattice attached to the spinners is characterised by $\varepsilon=0.2$. To generate an 


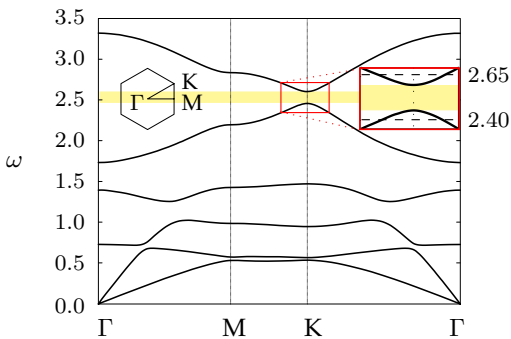

(a)

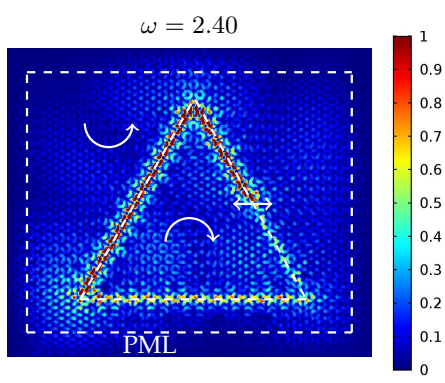

(b)

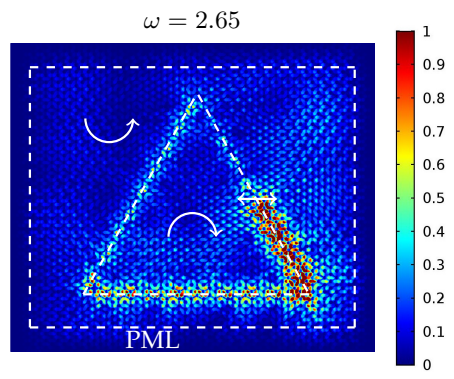

(c)

Fig. 2: (a) Dispersion diagram for the infinite inhomogeneous elastic system connected to spinners with $\alpha=0.7$ and $\varepsilon=0.2$. The response of the system with a triangular subdomain (indicated by dashed lines), where inside $\alpha=-0.7$ and outside $\alpha=0.7$, is shown in (b) and (c). The rotation directions of the spinners, according to these choices of $\alpha$, are indicated by the circular arrows. The absolute value of the displacement is shown for when the applied harmonic excitation has frequency (b) $\omega=2.40$ and $(c) \omega=2.65$. All other parameters are equal to unity.

interfacial waveform, we apply a harmonic displacement to a node along the internal boundary. The excitation frequencies chosen, $\omega=2.4$ and $\omega=2.65$, are situated in the immediate vicinity of the lower and upper limits, respectively, of the highest stop band (indicated by the yellow band in Figure 2(a)).

Figure 2(b) shows the response of the system when $\omega=2.40$. In this case, a localised waveform propagates along the triangular interface in the anti-clockwise direction. In Figure 2(c), when $\omega=2.65$, the direction of the waveform is reversed. In both cases, the intensity of the waveform decreases as it passes through vertices of the triangle, which create some scattering. It is clear from Figures 2(b) and (c) the waveforms have preferential directionality that is frequency dependent. We note that the directions of the waveforms may be reversed by interchanging the properties belonging to the spinners inside and outside the triangular subdomain.

\section{CONCLusions}

Here we have described a method for generating interfacial waveforms in an inhomogeneous elastic system connected to a non-uniform array of spinners. The waveforms propagate along closed paths defined by the boundaries of heterogeneities within the spinner arrangement. They also possess preferential directionality which can be controlled via the configuration of the spinners and the frequency of external excitation. It is anticipated this model will lead to new methods in the design of elastic structures for topological protection.

\section{ACKNOWLEDGEMENT}

M.J.N. gratefully acknowledges the support of the EU H2020 grant MSCA-IF-2016-747334-CAT-FFLAP. G.C., I.S.J., N.V.M. and A.B.M. thank the EPSRC (UK) for its support through Programme Grant no. EP/L024926/1.

\section{REFERENCES}

[1] M. Brun, I.S. Jones and A.B. Movchan: "Vortex-type elastic structured media and dynamic shielding," Proc. R. Soc. A, vol. 468, 3027-3046, 2012.

[2] G. Carta, M. Brun, A.B. Movchan, N.V. Movchan and I.S. Jones, "Dispersion properties of vortex-type monatomic lattices," Int. J. Solids Struct., vol. 51 2213-2225, 2014.

[3] P. Wang, L. Lu and K. Bertoldi, "Topological phononic crystals with one-way elastic edge waves," Phys. Rev. Lett. 115, $104302,2015$.

[4] L.M. Nash, D. Kleckner, A. Read, V. Vitelli, A.M. Turner and W.T.M. Irvine, "Topological mechanics of gyroscopic metamaterials," Proc. Natl. Acad. Sci., vol. 112, 14495-14500, 2015.

[5] G. Carta, I.S. Jones, N.V. Movchan, A.B. Movchan and M.J. Nieves, “"Deflecting elastic prism” and unidirectional localisation for waves in chiral elastic systems," Sci. Rep. 7, 26, 2017.

[6] M. Garau, G. Carta, M.J. Nieves, I.S. Jones, N.V. Movchan and A.B. Movchan: "Interfacial waveforms in chiral lattices with gyroscopic spinners," ArXiv: http://arxiv.org/abs/1802.10156. 